\title{
$\beta 1$ integrin modulates tumor growth and apoptosis of human colorectal cancer
}

\author{
JIA SONG, JIXIANG ZHANG, JING WANG, ZHUO CAO, JUN WANG, XUFENG GUO and WEIGUO DONG \\ Department of Gastroenterology, Renmin Hospital of Wuhan University, Wuhan, Hubei 430060, P.R. China
}

Received March 12, 2014; Accepted April 14, 2014

DOI: $10.3892 /$ or.2014.3168

\begin{abstract}
We aimed to ascertain whether $\beta 1$ integrin (ITGB1) induces apoptosis of colorectal cancer (CRC) through regulation of the mitochondrial pathway in vitro and in vivo. We generated lentiviral vectors expressing ITGB1 or ITGB1-specific RNAi and an unrelated control vector. After infection of the HT29 cells, we used western blot analysis and flow cytometric analysis to validate the patterns of ITGB1 expression. Proliferation and apoptosis were evaluated by colony formation assay, flow cytometry and western blot analysis. Upregulation of ITGB1 significantly increased the proliferation of HT29 cells; however, the levels of Bcl-2 and cyclin D1 proteins were upregulated while Bax, caspase-3, caspase-9 and p21 were downregulated in the HT29-ITGB1 cells compared to these levels in the controls. Hoechst 33258 staining and flow cytometric analysis showed that ITGB1 may play a significant role in the apoptosis of CRC cells. Moreover, ITGB1 promoted the proliferation of cells in a xenograft tumor mouse model. TUNEL staining revealed a marked increase in the percentage of positive cells in the HT29-RNAi group $(84.3 \pm 4.0 \%)$, which were more significant than in the HT29ITGB1 group $(48.3 \pm 2.9 \%)$ and the other two control groups, HT29 $(52.0 \pm 3.6 \%)$ and HT29-NC $(49.7 \pm 4.5 \%)$. These results suggest that ITGB1 regulates the growth and apoptosis of human colorectal cancer cells.
\end{abstract}

\section{Introduction}

Colorectal cancer (CRC) is one of the most common malignant diseases worldwide, and the prognosis remains poor $(1,2)$. The incidence of CRC has increased during recent decades, and the lifetime risk for CRC in industrialized countries is $\sim 5 \%$ (3). Early diagnosis and more effective treatment of $\mathrm{CRC}$ requires the identification of new biomarkers as well as insights into the molecular mechanisms of CRC.

Correspondence to: Professor Weiguo Dong, Department of Gastroenterology, Renmin Hospital, Wuhan University, Jiefang Road 238, Wuhan, Hubei 430060, P.R. China

E-mail:dwg@whu.edu.cn

Key words: integrin, colorectal cancer, apoptosis, proliferation
Integrins are heterodimeric transmembrane receptors for ligands in the extracellular matrix. Integrins contain two distinct chains called the $\alpha$ and $\beta$ subunits. In mammals, $18 \alpha$ - and $8 \beta$-subunits have been characterized. Integrins are essential for both cell adhesion and activation of intracellular signaling pathways (4). Each integrin generally consists of a non-covalently linked $\alpha$ - and $\beta$-subunit, with each subunit having a large extracellular domain, a single membrane-spanning domain and a short, non-catalytic cytoplasmic tail (5). These subunits combine to form 24 distinct heterodimers. Splice variants have been identified for some subunits and the number can reach at least 100 types $(6,7)$. Integrin signaling plays a key role in the regulation of cellular growth and tumor progression through the modulation of gene expression, apoptosis, cell adhesion, proliferation and migration (8). $\beta 1$ integrin (ITGB1) is the integrin mainly expressed in normal cells and in tumor-associated cells, where they control various developmental processes including angiogenesis, tumor progression, apoptosis and metastasis (9-14). Currently, the molecular mechanisms underlying the transcriptional regulation of ITGB1 expression in CRC are still not fully understood.

In the present study, we generated a lentivirus expressing ITGB1 or ITGB1-specific RNAi and an unrelated control and evaluated their effects on the human colorectal cancer cell line HT29. The ITGB1 expression, cell proliferation and cell apoptosis after infection were observed.

\section{Materials and methods}

Cells and animals. The human CRC cell line HT29 was purchased from the Shanghai Cell Collection (Shanghai, China) and maintained in Dulbecco's modified Eagle's medium (DMEM) (Gibco, Carlsbad, CA, USA) containing $10 \%$ fetal bovine serum (FBS; Gibco) at $37^{\circ} \mathrm{C}$ in a humidified atmosphere with $5 \% \mathrm{CO}_{2}$.

Lentivirus packaging. Three precursor miRNA (pre-miRNA) sequences targeting to ITGB1 (GenBank accession no. NM_133376) were designed using an Internet application system (Invitrogen). Double-stranded oligonucleotides encoding pre-miRNA sequence were annealed and inserted into the lentiviral vector GV248 (Shanghai GeneChem Co., Ltd., Shanghai, China). Real-time PCR and western blot analysis showed that the SR1 yielded the best suppression efficiency (data not shown); therefore, this construct was chosen 
to package the recombinant lentiviral vector for ITGB1 RNAi with the GV248 expression vector. Human ITGB1 was inserted into the GV218 lentiviral vector (Shanghai GeneChem) to upregulate expression of ITGB1. These two lentiviral vectors possessed a high level of expression of green fluorescent protein (GFP) and an antipuromycin site.

Lentiviral infection. The lentiviral vectors were transfected into HT29 cells with a multiplicity of infection (MOI) from 1 to 100 in the presence of $5 \mu \mathrm{g} / \mathrm{ml}$ polybrene (Sigma-Aldrich, St. Louis, MO, USA). To produce a stable transfected cell line, the cells were cultured in a selection medium containing $2.5 \mu \mathrm{g} / \mathrm{ml}$ of puromycin (Sigma-Aldrich) for 2 weeks. The transfected cells were named HT29-NC, HT29-RNAi and HT29-ITGB1.

Colony formation assay. For the colony formation assay, following treatment, adherent HT29, HT29-NC, HT29-RNAi and HT29-ITGB1 cells were trypsinized, and 1,000 viable cells (depending on the experiment) were subcultured in 6 -well plates (in triplicate). Cells were allowed to adhere and colonize for 14 days. To visualize the colonies, the medium was removed, and the cells were fixed in $96 \%$ ethanol for $10 \mathrm{~min}$ and stained with crystal violet staining solution. Where possible, colonies were counted, and are presented as the mean number of colonies \pm SD from at least three independent experiments.

Hoechst 33258 staining for apoptotic cells. HT29, HT29-NC, HT29-RNAi or HT29-ITGB1 cells in exponential growth were placed at a final concentration of $1 \times 10^{5}$ cells/well in a 6 -well plate. The cells were subsequently fixed, washed three times with PBS, and stained with Hoechst 33258 (Beyotime Institute of Biotechnology, Nantong, China) according to the manufacturer's protocol. Apoptotic features were assessed by analyzing chromatin condensation and by staining the fragments under an inverted fluorescence microscope (Olympus).

Western blot analysis. Cell lysates were prepared with RIPA lysis buffer (Beyotime Institute of Biotechnology) with phenylmethylsulfonyl fluoride (PMSF; Beyotime Institute of Biotechnology). The cell extracted protein was subjected to SDS-PAGE, transferred to polyvinylidene difluoride membranes (Millipore, Billerica, MA, USA), and blocked with $5 \%$ non-fat milk and $0.05 \%$ Tween. The membranes were immunoblotted with the following rabbit polyclonal antibodies: anti-ITGB1 (9699), anti-Bcl2 (2870), anti-Bax (5023), anti-p21 (2947), anti-cyclin D1 (2978), anti-caspase-9 (9502), anti-caspase-3 (9662) or anti-GAPDH (2118) (all from Cell Signaling Technology, Beverly, MA, USA).

The blots were rinsed three times in TBST and incubated with a 1:10,000 diluted goat anti-rabbit secondary antibody (LI-COR Biosciences, Lincoln, NE, USA) for $1 \mathrm{~h}$ at room temperature before they were washed extensively with TBST. Finally, the membranes were scanned with a two-color infrared imaging system (Odyssey, Lincoln, NE, USA). Membranes were also probed for GAPDH as an additional loading control.

Flow cytometric analysis of cell apoptosis. The HT29, HT29NC, HT29-RNAi and HT29-ITGB1 cells were washed with
PBS, centrifuged at 12,000 $\mathrm{x}$ g for $5 \mathrm{~min}$ and then resuspended in $500 \mu \mathrm{l}$ binding buffer. Double staining with Annexin V-PE and 7-ADD was performed using the Annexin V-FITC kit (Beyotime Institute of Biotechnology) according to the manufacturer's recommendations, and the cells were then analyzed by FACS (BD FACSAria III; BD Biosciences, San Jose, CA, USA). The data were analyzed using FlowJo 7.6 software (Tree Star Inc., Ashland, OR, USA).

Xenograft tumor model. Male nude mice (4-6 weeks of age, $\mathrm{n}=24$ ) (Beijing HFK Bioscience Co., Ltd., Beijing China) were used in the experiments. After alcohol preparation of the skin, $1 \times 10^{6}$ HT29, HT29-NC, HT29-RNAi or HT29-ITGB1 cells suspended in $100 \mu \mathrm{l}$ PBS, were subcutaneously inoculated into the dorsal area of the nude mice. At the end of the experiment, tumors were harvested and weighed. All experiments were performed according to the recommendations of the Institutional Animal Care and Use Committee, and the study protocol was approved by the Ethics Committee for Animal Research of Wuhan University, China.

TdT-mediated dUTP-biotin nick end-labeling assay. The TUNEL technique was performed to detect and quantitate apoptotic cell death using the In Situ Cell Death Detection kit (Roche Diagnostics Corporation, Indianapolis, IN, USA) following the provided instructions. Briefly, chamber slides were fixed with $4 \%$ paraformaldehyde and permeabilized in $0.1 \%$ Triton $\mathrm{X}-100$. The slides were then incubated with TUNEL reaction mixture for $1 \mathrm{~h}$ at $37^{\circ} \mathrm{C}$. After washing with PBS, the slides were incubated with peroxidase-conjugated antibody for $30 \mathrm{~min}$ at $37^{\circ} \mathrm{C}$ and were developed with the DAB system. Under microscopy, 6 fields were randomly selected from every sample and 100 cells were randomly selected from every field. The apoptotic rate $=$ (number of total apoptotic cells/100) x $100 \%$.

Statistical analysis. Data are expressed as the mean \pm SD. The difference among groups was determined by ANOVA, and the difference between groups was analyzed by the Student's t-test using SPSS 17.0 for Microsoft Windows (SPSS Inc., Chicago, IL, USA). A value of $\mathrm{P}<0.05$ was considered to indicate a statistically significant result.

\section{Results}

Increased transduction of the lentiviral vector in vitro and in vivo. EGFP was used as a marker to detect whether the recombinant lentiviral vectors were successfully transduced in vitro and in vivo. The transfection efficiency (Fig. 1) in vitro approached $95 \%$ and in vivo the efficiency was at least $90 \%$.

Lentiviral-mediated ITGB1 expression in HT29 cells. Western blot analyses revealed that the level of the ITGB1 transcripts in the HT29-ITGB1 cells was significantly increased $(\mathrm{P}<0.05)$. The level of ITGB1 transcripts in the HT29-RNAi cells was decreased compared to the level in the HT29 and HT29-NC cells $(\mathrm{P}<0.05)$. As expected, infection with the control lentivirus did not affect the expression of ITGB1 (Fig. 2).

In addition, as shown in Fig. 2, transfection efficiency was detected by flow cytometric analysis. The transfection rate 


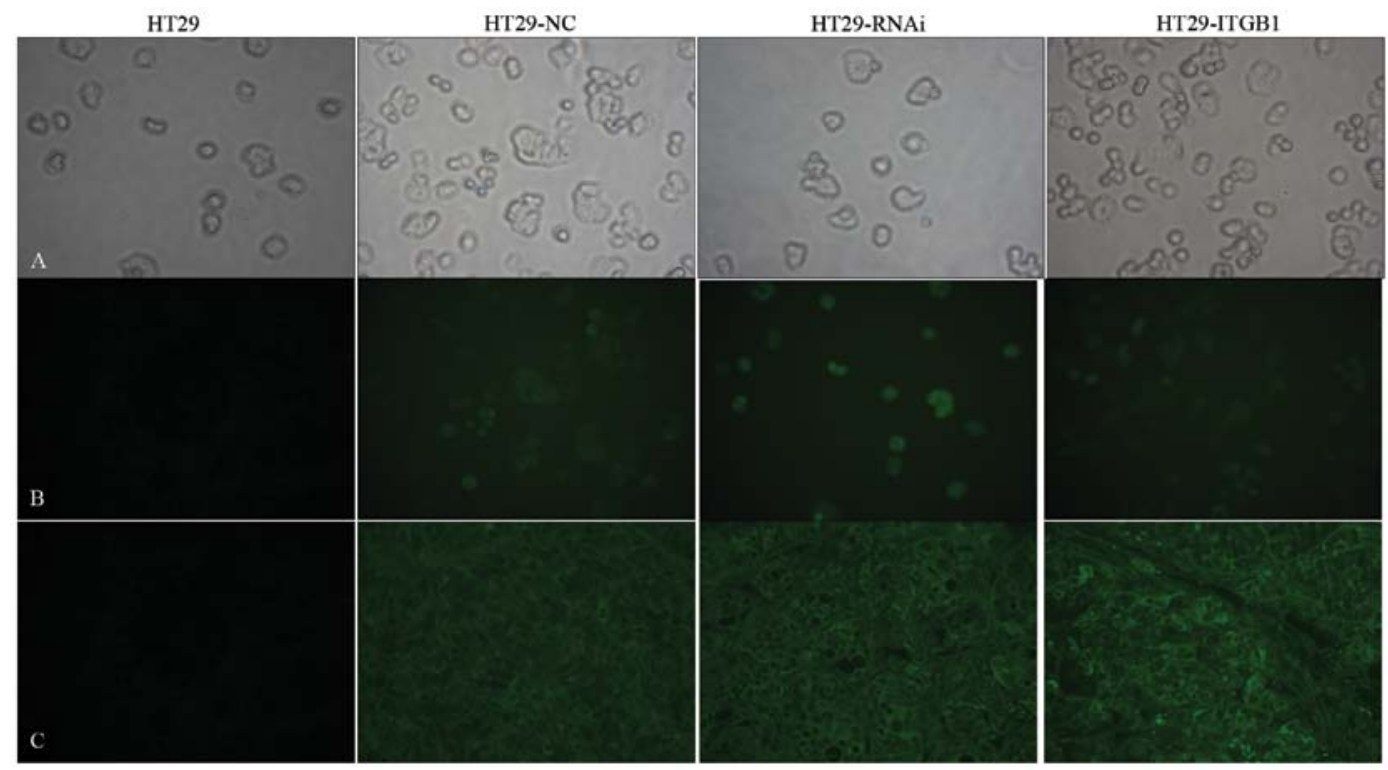

Figure 1. Fluorescent expression in vitro and in vivo. (A) Light microphotographs of HT29-NC, HT29-RNAi and HT29-ITGB1 cells (original magnification, x200). (B) Green fluorescent protein (GFP) was detected under a fluorescence microscope in HT29-NC, HT29-RNAi and HT29-ITGB1 cells (original magnification, $\mathrm{x} 200$ ). (C) Fluorescent expression in the xenograft tumors (original magnification, $\mathrm{x} 400$ ).

A

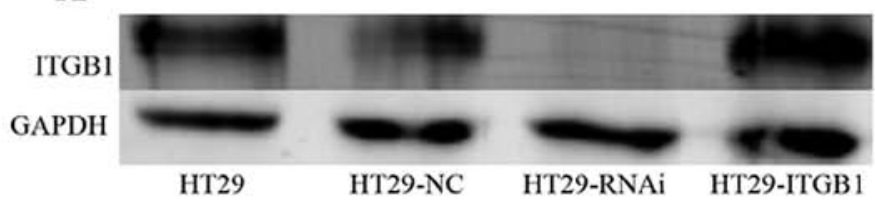
ITGB1 protein levels in the HT29, HT29-NC, HT29-RNAi and HT29-ITGB1 cells ( $\left.{ }^{*}<0.05\right)$. (B) Flow cytometric assay shows the transfection efficiency in the HT29-NC, HT29-RNAi and HT29-ITGB1 cells $\left(\mathrm{P} 2, \mathrm{GFP}^{+}\right)$.
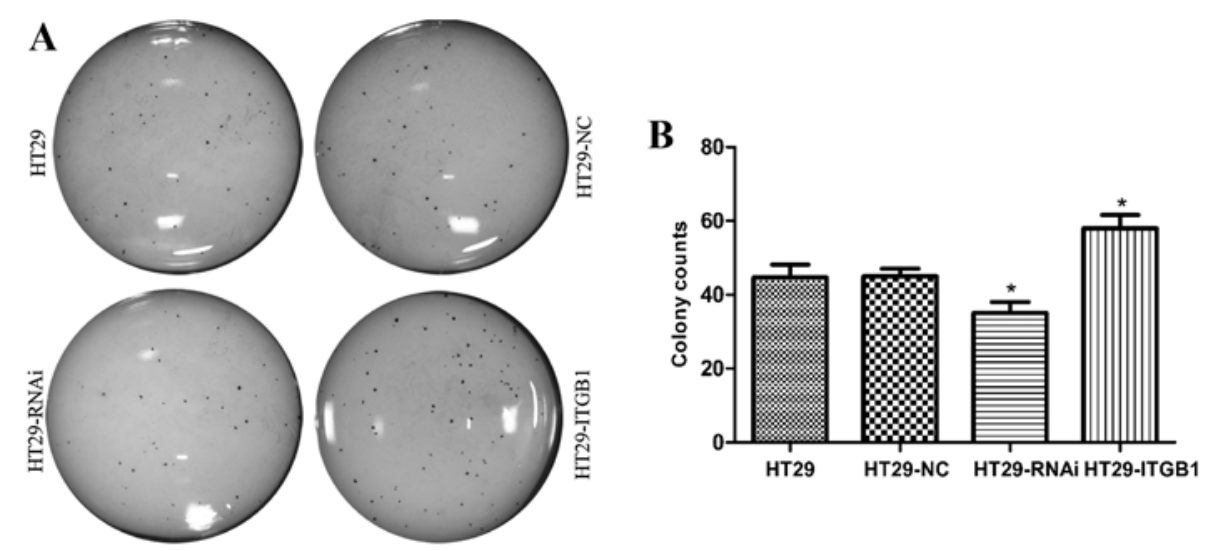

Figure 3. Overexpression of ITGB1 induces proliferation in human colorectal cancer HT29 cells. (A) ITGB1 inhibited tumor cell colony formation. (B) Quantitative analysis of colony numbers is represented as the mean \pm SD. "P<0.05, compared to the HT29 and HT29-NC cells.

was $90.2,93.8$ and $94.8 \%$ in the HT29-NC, HT29-RNAi and HT29-ITGB1 cells, respectively.

Effect of ITGB1 expression on the proliferation of HT29 cells. To determine the effect of ITGB1 expression on the growth of
HT29 cells, the proliferation of HT29, HT29-NC, HT29-RNAi and HT29-ITGB1 cells was determined by clone formation assay (Fig. 3). Following incubation for 2 weeks, $45 \pm 3.5$, $45 \pm 2.0,35 \pm 3.0$ and $58 \pm 3.6$ colonies were generated from the HT29, HT29-NC, HT29-RNAi and HT29-ITGB1 cells, respec- 


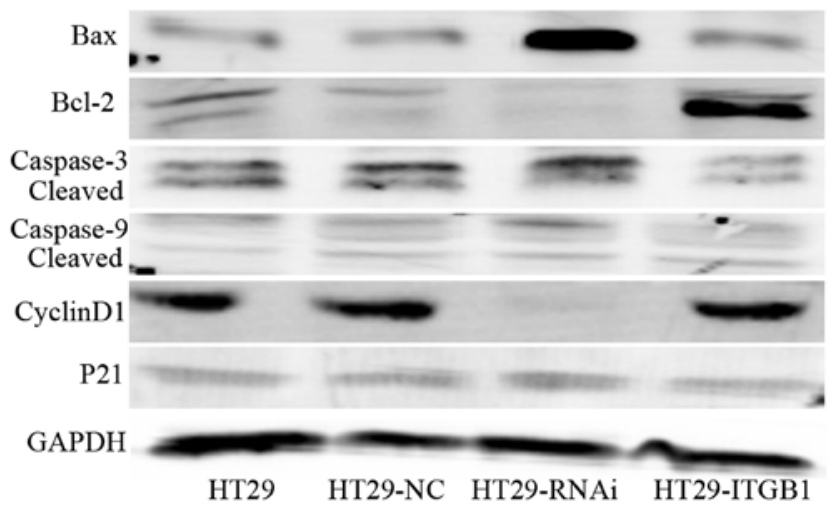

Figure 4. Western blots showing the expression of apoptosis-related proteins in vitro. The levels of Bcl-2, Bax, caspase-3, caspase-9, cyclin D1 and p21 proteins in the HT29, HT29-NC, HT29-RNAi and HT29-ITGB1 cells. GAPDH expression was used as internal control. A representative blot is shown from three independent experiments with identical results.
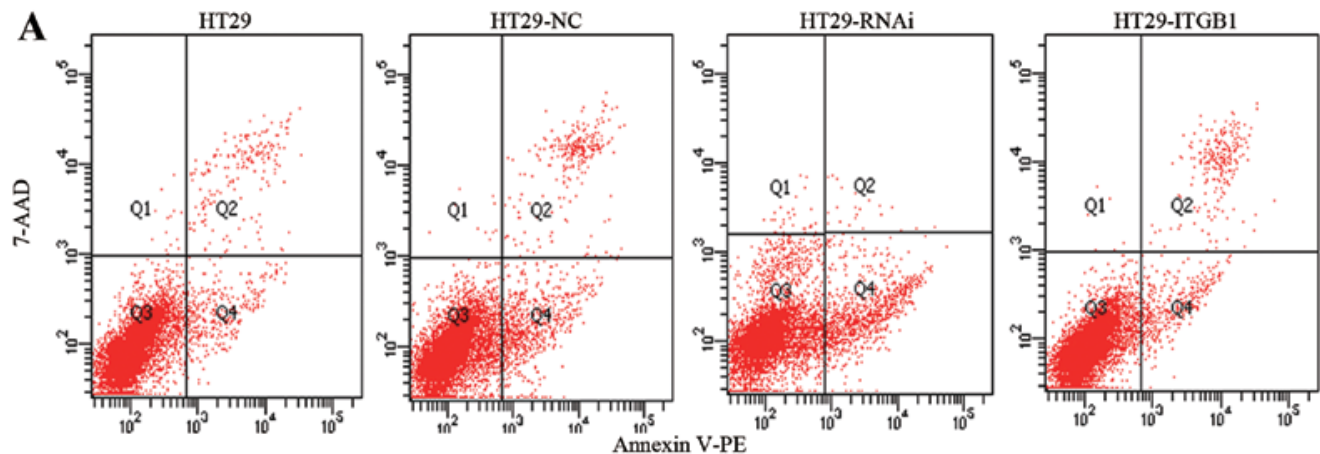

C

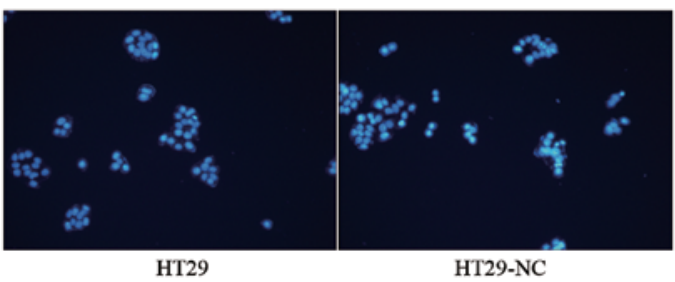

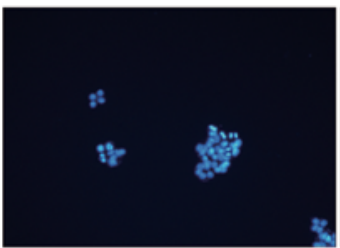

HT29-RNAi

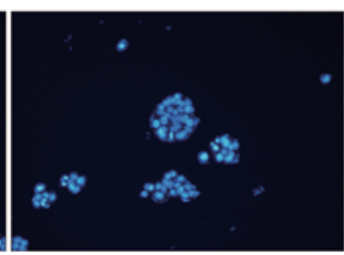

HT29-ITGB1
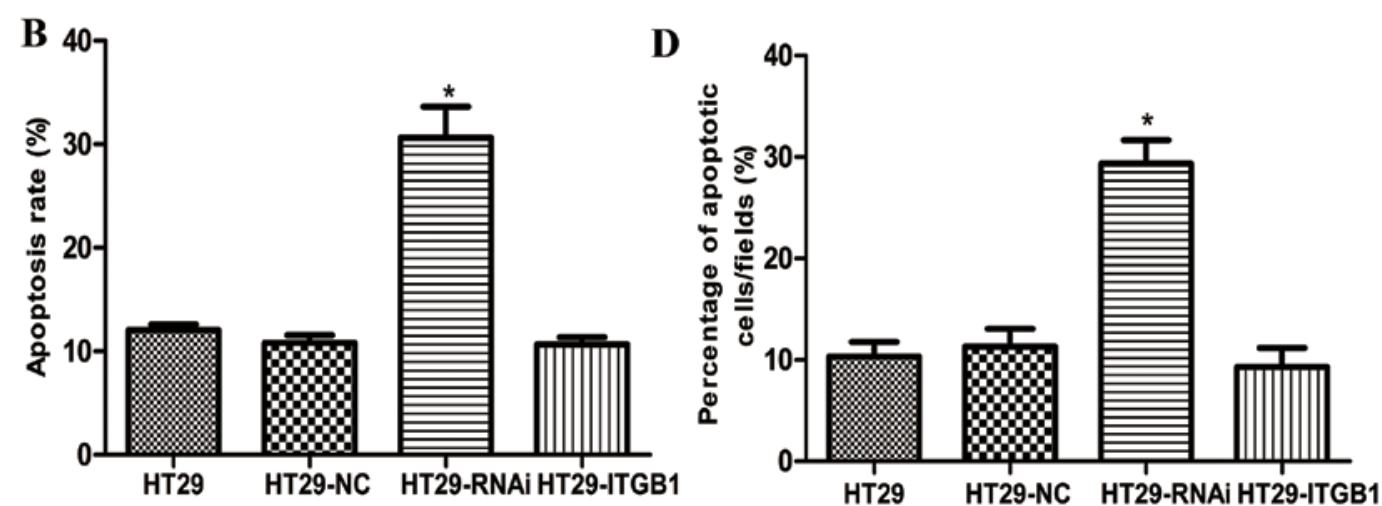

Figure 5. Effect of ITGB1 expression on cell apoptosis. (A) Detection of apoptosis via Annexin V-PE/7-AAD staining. The proportion of non-apoptotic cells (Q3, Annexin V-PE/7-AAD), early apoptotic cells (Q4, Annexin V-PE $\left.+7-\mathrm{AAD}^{-}\right)$, late apoptotic/necrotic cells (Q2, Annexin V-PE /7-AAD $)$and cell debris or death cell (Q1). (B) Data shown are means \pm SD from three independent experiments. (C) Apoptosis was assessed using Hoechst 33258, and apoptotic features were assessed by observing chromatin condensation and fragment staining (original magnification, $\mathrm{x} 200$ ). (D) Quantitative analysis of apoptotic cells is represented as the mean \pm SD from three independent experiments. " $\mathrm{P}<0.05$, compared to the HT29 and HT29-NC cells.

tively. The HT29-ITGB1 cells showed increased proliferation when compared to the HT29 and HT29-NC cells. In contrast, the proliferation of HT29-RNAi cells was much slower as compared with the other two control groups $(\mathrm{P}<0.05)$. 


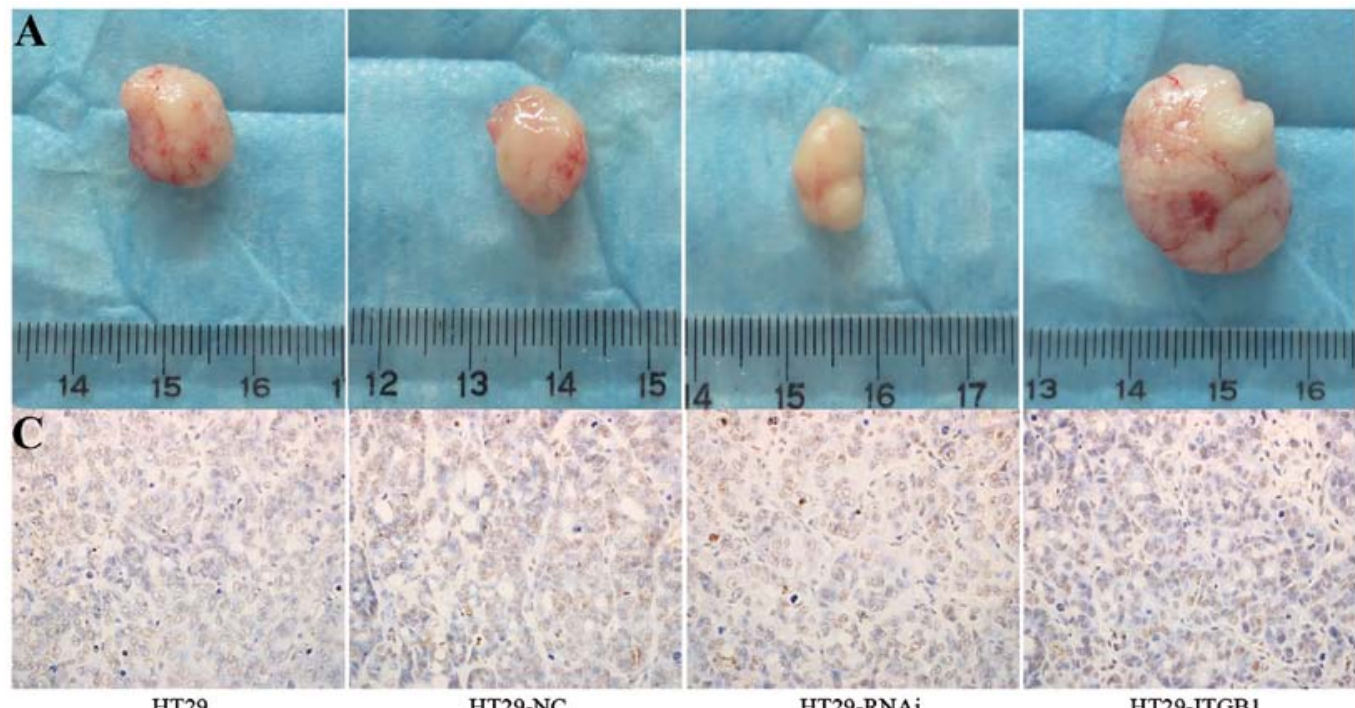

B

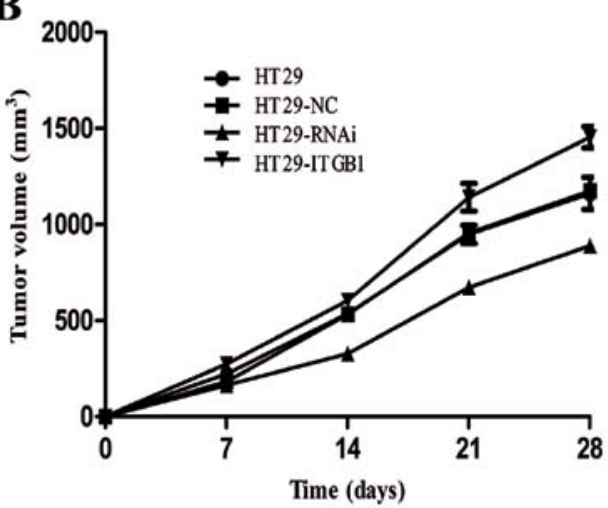

HT29-RNAi

D 100

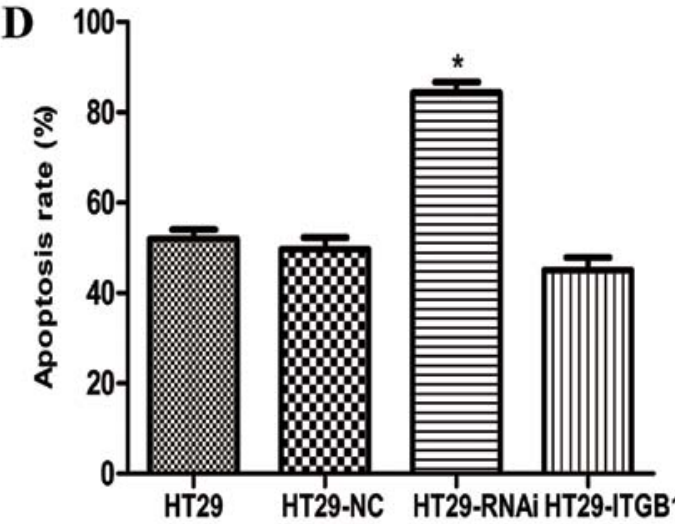

Figure 6. Effects of ITGB1 on mouse xenograft tumors in vivo. (A) Cells were injected subcutaneously into nude mice. One group of mice received HT29, HT29-NC, HT29-RNAi or HT29-ITGB1 cells while a control group of mice received PBS. Representative images of a tumor in each group post inoculation. (C) Apoptotic cells, shown by TUNEL staining, were significantly increased in the HT29-RNAi group (original magnification, x400). (B) Tumor volumes of the xenograft tumors derived from HT29, HT29-NC, HT29-RNAi or HT29-ITGB1 cells. (D) Quantitative analysis of apoptotic cells in the HT29, HT29-NC, HT29-RNAi and HT29-ITGB1 groups. Data are presented as mean \pm SD. ${ }^{~} \mathrm{P}<0.05$, compared to the HT29 and HT29-NC groups.

ITGB1 inhibits expression of apoptosis-related proteins in HT29 cells. We assessed the effects of Bcl-2, Bax, caspase-3, caspase- 9 , cyclin $\mathrm{D} 1$ and $\mathrm{p} 21$ protein by western blotting in the HT29, HT29-NC, HT29-RNAi and HT29-ITGB1 cells. The levels of Bcl-2 and cyclin D1 proteins were upregulated while Bax, caspase-3, caspase- 9 and p21 levels were downregulated in the HT29-ITGB1 cells when compared to the controls (Fig. 4). However, the results were found to be opposite in the HT29-RNAi cells. Taken together, these findings indicate that ITGB1 activates the mitochondrial signaling pathway in human HT29 cells.

Effect of ITGB1 on HT29 cell apoptosis. By using flow cytometric analysis, we assessed the effect of ITGB1 on HT29 cell apoptosis. HT29, HT29-NC, HT29-RNAi and HT29-ITGB1 cells were incubated with Annexin V-PE in a buffer containing 7-AAD and were analyzed by flow cytometric analysis.

The results in Fig. 5 show that the HT29-RNAi cells exhibited a higher apoptosis rate up to $30.6 \pm 5.1 \%(\mathrm{P}<0.05)$ when compared with the HT29 $(12.1 \pm 0.9 \%)$ and HT29-NC cells $(10.8 \pm 1.3 \%)$. However, the HT29-ITGB1 cells had an apoptosis rate of only $12.3 \pm 2.5 \%$, and as expected, no difference was noted between the HT29 and HT29-NC cells $(\mathrm{P}>0.05)$.

Antitumor efficacy of ITGB1 in nude mice. On the basis of the in vitro data above, we investigated the effects of lentivirus treatment on tumor growth in vivo. The development of solid HT29 tumors was monitored for 30 days. As shown in Fig. 6, solid tumors were first visible $\sim 5$ days post inoculation and grew rapidly afterwards in the HT29-ITGB1 group $\left(1454 \pm 95.65 \mathrm{~mm}^{3}\right)$. In contrast, the HT29-RNAi group had a slower solid tumor growth rate and a smaller mean tumor volume $\left(889.3 \pm 61.49 \mathrm{~mm}^{3}\right)$ compared to the control groups $\left(1162 \pm 144.8\right.$ and $\left.1174 \pm 58.41 \mathrm{~mm}^{3} ; \mathrm{P}<0.05\right)$ (Fig. 6A and B). These results indicate that HT29-ITGB1 cells have high tumorigenicity following inoculation in immunocompromised nude mice.

Apoptotic cells in the tumor sections were analyzed by TUNEL staining. TUNEL staining showed markedly more positive cells in the HT29-RNAi group $(84.3 \pm 4.0 \%)$, which were more significant than in the HT29-ITGB1 group $(48.3 \pm 2.9 \%)$ and the other two control groups, HT29 $(52.0 \pm 3.6 \%)$ and HT29-NC (49.7 $\pm 4.5 \%)$ (Fig. 6C and D). 


\section{Discussion}

ITGB1 is the most widely expressed integrin in cells and it has been suggested to play a role in predicting the clinical course and prognosis of several types of cancers $(15,16)$. A number of studies suggest that ITGB1 is important but not essential for metastasis in different types of cancer $(17,18)$. In another study ITGB1 inhibition was found to induce apoptosis in breast cancer cells (19). The current series of experiments demonstrated the essential role of ITGB1 in maintaining cell survival and promoting tumorigenesis in cancer cells. In the present study, we generated a lentivirus expressing ITGB1 or ITGB1-specific RNAi and an unrelated control and evaluated their effects on the human colorectal cancer cell line HT29. We found that upregulation of ITGB1 expression greatly induced proliferation of HT29 cells in vitro, as shown by higher proliferation efficiency in HT29-ITGB1 cells compared to controls. In addition, the average volume of tumors in the HT29-ITGB1 group was significantly increased compared to that of the other three groups. In the present study, we found a relationship between expression of ITGB1 and proliferation. However, the mechanism(s) underlying the action of ITGB1 and the downstream effects on the ITGB1 target genes remain unclear.

Cell proliferation and apoptosis are two essential conditions for cell transformation and malignant tumorigenesis. The B-cell leukemia/lymphoma 2 (Bcl-2) family plays a pivotal role in the control of apoptosis and can be classified into two functionally distinct groups: anti-apoptotic proteins and proapoptotic proteins (20). Bcl-2, an anti-apoptotic member of the $\mathrm{Bcl}$ family, was initially identified as a proto-oncogene detected in human B-cell follicular lymphoma (21). Bcl-2-associated X protein (Bax), a pro-apoptotic protein is expressed abundantly and selectively during apoptosis and promotes cell death (22). An increased ratio of Bcl-2 to Bax has commonly been used to determine the induction of apoptosis in a number of tissue types (23).

In the present study, we explored whether ITGB1 modulates the apoptosis of HT29 cells in vitro and found that HT29-RNAi cells underwent rapid apoptosis when compared with the other groups. Therefore, we conclude that downregulation of ITGB1 expression markedly enhances the apoptosis capacity of HT29 cells in vitro. Western blot analysis showed that the level of Bcl-2 was upregulated while Bax, caspase-3 and caspase-9 levels were downregulated in the HT29-ITGB1 cells when compared to the controls. The significant effect of ITGB1 on HT29 cells suggests that ITGB1 may play an important role in the apoptosis of CRC cells.

Cell cycle progression is likely to play an important role in gastrointestinal carcinogenesis. Cell cycle progression is governed by a family of cyclin-dependent kinases (CDKs), which are activated by binding to cyclin proteins and inhibited by CDK inhibitors (24). Cyclin D1 is a key regulator of the G1 phase of the cell cycle and is located on chromosome 11q13. A significant proportion of dysplasias contain molecular abnormalities that may result in cyclin D1 overexpression (25). Our data showed that ITGB1 upregulated cyclin D1 protein while the p21 level was downregulated in the HT29-ITGB1 cells when compared with the other groups. This indicates that ITGB1 may participate in the cell cycle regulation of CRC cells, but the mechanism(s) underlying the action of ITGB1 remain unclear.

Taken together, based on our data, we demonstraed that ITGB1 may regulate the growth and apoptosis of HT29 cells both in vitro and in vivo. Our findings extend our knowledge concerning the pathogenesis of CRC and may assist in developing therapies for human CRC.

\section{Acknowledgements}

The present study was supported by research grants from the National Natural Science Foundation of China (no. 81172069) and the Science and Technology Department of Hubei Province (no. 2010CDA043).

\section{References}

1. Zheng S and Shanrong C: Colorectal cancer epidemiology and prevention study in China. Chin Ger J Clin Oncol 2: 72-75, 2003.

2. Yang L, Parkin DM, Ferlay J, Li L and Chen Y: Estimates of cancer incidence in China for 2000 and projections for 2005. Cancer Epidemiol Biomarkers Prev 14: 243-250, 2005.

3. Jemal A, Siegel R, Xu J and Ward E: Cancer statistics, 2010. CA Cancer J Clin 60: 277-300, 2010.

4. Hynes RO: Integrins: bidirectional, allosteric signaling machines. Cell 110: 673-687, 2002.

5. Hood JD and Cheresh DA: Role of integrins in cell invasion and migration. Nat Rev Cancer 2: 91-100, 2002.

6. Melker AA and Sonnenberg A: Integrins: alternative splicing as a mechanism to regulate ligand binding and integrin signaling events. Bioessays 21: 499-509, 1999.

7. Gilcrease MZ: Integrin signaling in epithelial cells. Cancer Lett 247: 1-25, 2007.

8. Felding-Habermann B: Integrin adhesion receptors in tumor metastasis. Clin Exp Metastasis 20: 203-213, 2003.

9. Li N, Zhang Y, Naylor MJ, Schatzmann F, Maurer F, Wintermantel T, Schuetz G, Mueller U, Streuli CH and Hynes NE: $\beta$ integrins regulates mammary proliferation and maintain the integrity of mammary alveoli. EMBO J 24: 1942-1953, 2005.

10. Arao S, Masumoto A and Otsuki M: $\beta 1$ integrins play an essential role in adhesion and invasion of pancreatic carcinoma cells. Pancreas 20: 129-137, 2000.

11. Aumailley M, Pesch M, Tunggal L, Gaill F and Fässler R: Altered synthesis of laminin 1 and absence of basement membrane component deposition in $\beta 1$ integrin deficient embryoid bodies. J Cell Sci 113: 259-268, 2001.

12. Stroeke PJ, van Rijthoven EA, Boer E, Geerts D and Roos E: Cytoplasmic domain mutants of $\beta 1$ integrin, expressed in $\beta 1$-knockout lymphoma cells, have distinct effects on adhesion, invasion and metastasis. Oncogene 19: 1232-1238, 2000.

13. Brakebusch $\mathrm{C}$ and Fassler R: $\beta 1$ integrin function in vivo: adhesion, migration and more. Cancer Metastasis Rev 24: 403-411, 2005.

14. Wang W, Goswami S, Lapidus K, Wells AL, Wyckoff JB, Sahai E, Singer RH, Segall JE and Condeelis JS: Identification and testing of a gene expression signature of invasive carcinoma cells within primary mammary tumors. Cancer Res 64: 8585-8594, 2004.

15. Yao ES, Zhang H, Chen YY, Lee B, Chew K, Moore D and Park C: Increased $\beta 1$ integrin is associated with decreased survival in invasive breast cancer. Cancer Res 67: 659-664, 2007.

16. Wang D, Müller S, Amin AR, Huang D, Su L, Hu Z, Rahman MA, Nannapaneni S, Koenig L, Chen Z, Tighiouart M, Shin DM and Chen ZG: The pivotal role of integrin $\beta 1$ in metastasis of head and neck squamous cell carcinoma. Clin Cancer Res 18: 4589-4599, 2012.

17. Adachi M, Taki T, Higashiyama M, Kohno N, Inufusa $\mathrm{H}$ and Miyake M: Significance of integrin $\alpha 5$ gene expression as a prognostic factor in node-negative non-small cell lung cancer. Clin Cancer Res 6: 96-101, 2000. 
18. Ahmed N, Riley C, Rice G and Quinn M: Role of integrin receptors for fibronectin, collagen and laminin in the regulation of ovarian carcinoma functions in response to a matrix microenvironment. Clin Exp Metastasis 22: 391-402, 2005.

19. Park CC, Zhang H, Pallavicini M, Gray JW, Baehner F, Park CJ and Bissell $\mathrm{MJ}: \beta 1$ integrin inhibitory antibody induces apoptosis of breast cancer cells, inhibits growth and distinguishes malignant from normal phenotype in three dimensional cultures and in vivo. Cancer Res 66: 1526-1535, 2006.

20. Cory S and Adams JM: The Bcl 2 family: regulators of the cellular life-or-death switch. Nat Rev Cancer 2: 647-656, 2002.

21. Bakhshi A, Jensen JP, Goldman P, Wright JJ, McBride OW Epstein AL and Korsmeyer SJ: Cloning the chromosomal breakpoint of $\mathrm{t}(14 ; 18)$ human lymphomas: clustering around $\mathrm{JH}$ on chromosome 14 and near a transcriptional unit on 18. Cell 41: 899-906, 1985
22. Puthalakath $\mathrm{H}$ and Strasser A: Keeping killers on a tight leash: transcriptional and post-translational control of the pro-apoptotic activity of BH3-only proteins (Review). Cell Death Differ 9: 505-512, 2002.

23. Thome M and Tschopp J: Regulation of lymphocyte proliferation and death by FLIP. Nat Rev Immunol 1: 50-58, 2001.

24. Sherr CJ: G1 phase progression: Cycling on cue. Cell 79: 551-555, 1994.

25. Huang S, Chen CS and Ingber DE: Control of cyclin D1, p27 ${ }^{\mathrm{Kip} 1}$ and cell cycle progression in human capillary endothelial cells by cell shape and cytoskeletal tension. Mol Biol Cell 9: 3179-3193, 1998. 